
3 Research Square
Preprints are preliminary reports that have not undergone peer review.
They should not be considered conclusive, used to inform clinical practice, or referenced by the media as validated information.

\title{
The Relationship Between Erythrocyte Membrane Fatty Acid Content and Serum Adiponectin Level in Military Personnel, a Cross Sectional Study
}

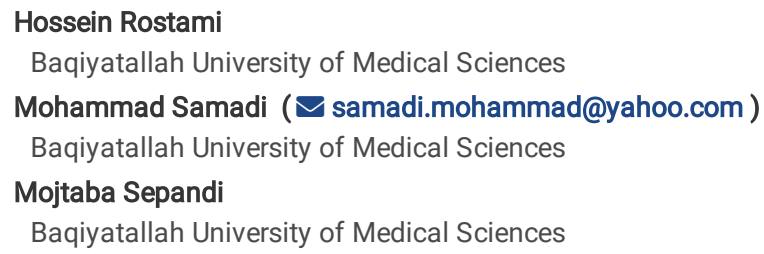

\section{Research}

Keywords: Cardiovascular disease, military personnel, serum adiponectin, dietary fatty acid, erythrocyte membrane fatty acid, serum lipids

Posted Date: May 6th, 2021

DOI: https://doi.org/10.21203/rs.3.rs-482275/v1

License: () (1) This work is licensed under a Creative Commons Attribution 4.0 International License. Read Full License 


\section{Abstract}

Background: Military personnel compared to civilian people are more prone to cardiovascular disease (CVD) however the relationship between dietary fatty acid intake and CVD markers is not well established in them. The aim of this study was to investigate the relationship between dietary fatty acid intake and erythrocyte membrane fatty acid content as well as serum adiponectin and lipid profile in military personnel.

Method: Four hundred military personnel were recruited from the population of army individuals in Iran. The dietary fatty acid intake was determined from a food frequency questionnaire (FFQ) completed by each military personnel. Fasting blood samples were also obtained from each individual and fatty acids derived from erythrocyte membrane, serum lipid profile and adiponectin levels were measured. The correlation between dietary fatty acid intake and biochemical parameters were analyzed by univariate regression analysis.

Results: There was a negative significant association between total saturated fatty acid, lauric, myristic, palmitic acid and a positive association between eicosapentanoic acid and docosahexanoic acid in erythrocyte-membrane with adiponectin level. Moreover, we found that dietary SFA intake had a significant negative correlation with serum adiponectin level. In addition, dietary SFA intake showed a significant positive correlation with serum total cholesterol and LDL-c levels. In contrary, dietary PUFA and MUFA intake revealed a significant inverse association with serum cholesterol level and LDL-c.

Conclusion: We concluded that dietary SCFAs might be associated with lower serum adiponectin and higher cholesterol levels, and suggest that CVD risk factor might be suppressed, by reducing dietary SCFA intake.

\section{Introduction}

Nutrition in military personnel has been always a challenging issue. Without proper nutrition, it cannot be expected that military forces be able to play a major role in defending the country's borders and sovereignty [1]. To ensure that the physical capacity and mental performance of military personnel remain at the desired level, the nutrition of military personnel should be balanced and optimum in quality and quantity [2]. Recently, it has been found that a significant proportion of soldiers in military operations do not consume nutritionally balanced diet, which leads to lose their physical stamina and efficiency [3]. Military forces, who participate in military operations, need more energy, micronutrients and macronutrients than administrative personnel [4].

On the other hand, overweight and obesity in army individuals not only affects the operational capabilities but also affect their health and wellbeing [5]. Some studies have shown an association between overweight, obesity and cardiovascular diseases (CVDs) and over nutrition is one of the major causal factors in this regard [6]. A number of studies from several countries including one from Iran reported the prevalence of CVDs in military personnel [7]. However, it has been reported that military personnel have a number of work related stress [8] when work-related stress has been reported as one of the potential risk factors for CVD [9]. It has been also reported that there is a higher rate of chronic disease including CVD [10] in veterans of the military personnel compared to civilian people in the United States. Additionally, participating in war [11] and recovery from war related traumatic health issues [12] have been led to increase the risk and episodes of CVD and cardiovascular events and the risk of development of CVD increases with the severity of injury [13]. Consequently, it is assumed that military personnel are more susceptible to CVD compared with civilian people.

Some risk factors of CVD are traditional such as high level of LDL-cholesterol (LDL-c), low level of HDL-cholesterol (HDL-c), elevated level of triglyceride (TG), diabetes, blood pressure, obesity, oxidative stress and inactivity [14]. More recently, adiponectin, an anti-inflammatory biomarker, has been found as a major biomarker for the early detection of CVDs [15]. According to the guidelines of adult treatment panel III (ATP III), dyslipidemia is the main risk factor for CVD and decreasing the dietary saturated fatty acid (SFA) intake lower than $10 \%$ of calorie intake regularly, declines the risk of CVD [14]. Furthermore, a previous study reported the positive effect of polyunsaturated fatty acids (PUFA) and monounsaturated fatty acids (MUFA) in reducing serum LDL-c level in experimental animal [16]. Some reports also have shown that intake of n-3 polyunsaturated fatty acids (n-3 PUFAs) decrease the level of serum TG in human subjects [17]. However, sufficient studies have not been conducted in this regard in Iranian military personnel.

With regard to the scarcity of study in Iranian military personnel in relation to the effect of dietary parameters on the risk factors of CVD, we intended to explore the relationship between erythrocyte-membrane fatty acids in addition to dietary fatty acids intake with serum adiponectin and serum lipid levels.

Our study has been designed to overcome some biases as observed in some previous epidemiological studies [18]. In epidemiological studies, food frequency questionnaire (FFQ) was applied to evaluate the dietary fatty acid intake [19]. However, estimation of fat intake in FFQ was prone to subjective biases such asinaccurate memories, refusal and incapability to provide detail dietary components and insufficient knowledge of measuring scales. In addition, databases of nutrients may improperly reveal the changes in dietary intake [20]. Considering these biases, the different fatty acids in erythrocyte membrane were measured not only to validate our data but also to improve the quality of our study.

\section{Materials And Methods}

This observational cross-sectional study was conducted between July 2019 and May 2020. Considering all inclusion and exclusion criteria as given below, 400 eligible military personnel were recruited from the population of army individuals in south of Iran. The inclusion criteria to enter in the study were, willing to participate, being a permanent member of military services or being at least one year or more as a soldier, only men, eating at least two main course meals in camp and the exclusion criteria were: using drugs related to thyroid, heart disease, blood pressure, inflammation and if there is any medical history of inflammatory diseases. After explaining the goal of the study, the participants were invited to the laboratory of corresponding clinic in fasting state when an informed consent form was completed by each participating individual.

The research protocol was approved by ethic committee of Baqiyatallah University of Medical Sciences (IR.BMSU.REC.1398.056), Tehran, Iran and it was conducted according to the ethical guidelines of the Helsinki declaration.

Page 2/13 


\section{Intervention}

Data were gathered during 9-month long intervention trial. A validated food frequency questionnaire (FFQ) was obtained from each participant and the level of the activities of participant was determined by the International Physical Activity Questionnaire.

\section{Measurements of serum parameters}

A $5 \mathrm{ml}$ of venous blood in fasting state was obtained from antecubital area of each individual between 7.00 and 8.00 0'clock in the morning. The blood samples were centrifuged at $3000 \mathrm{~g}$ for $15 \mathrm{~min}$ at $4^{\circ} \mathrm{C}$ and serum was separated. The serum samples were immediately transferred to the laboratory for the measurement of fasting plasma glucose (FPG), TG, total Cholesterol, HDL-c and adiponectin levels.

The FPG and serum total cholesterol, LDL-c and triglycerides were measured by an Auto Analyzer Selectra E kit (Pars Azmoon, Tehran, Iran). The serum LDL-c level was calculated by Friedewald equation as given below, using the data of total cholesterol and triglycerides when 1/5th of triglycerides (TG/5) were considered as the equivalence of very low density lipoprotein-cholesterol (VLDL-c).

Low density lipoprtoein-cholesterol $(\mathrm{LDL}-\mathrm{C})=[$ Total cholesterol $-(\mathrm{HDL}-\mathrm{C}+\mathrm{TG} / 5)]$

Serum adiponectin level was measured by a commercially available ELISA kit (AdipoGen, Incheon, Korea, Cat. No. AG-45A-0001EK-KI01). The limit of detection of the assay was $100 \mathrm{pg} / \mathrm{mL}$; the intra- and inter-assay coefficients of variance were $3.1 \%-3.7 \%$ and $2.5 \%-4.9 \%$, respectively.

The cut-off points for considering abnormalities were set at $>160 \mathrm{mg} / \mathrm{dL}$ for LDL-c, $<40 \mathrm{mg} / \mathrm{dL}$ for HDL-c, $>150 \mathrm{mg} / \mathrm{dL}$ for TG, $>200 \mathrm{mg} / \mathrm{dL}$ for total cholesterol, > $126 \mathrm{mg} / \mathrm{dL}$ for fasting plasma glucose.

\section{Measurements of fatty acid in erythrocyte membrane}

After an overnight fast for a period of 12-h, blood was collected from each individual in EDTA-containing tubes. The blood was centrifuged at $500 \mathrm{~g}$ for 15 minutes at room temperature. Then plasma was immediately separated from red blood cells and the sediment was diluted with the equal volume of $0.9 \% \mathrm{NaCl}$ solution to avoid red blood cell hemolyze and stored at $-18^{\circ} \mathrm{C}$ until further analysis. After gathering all samples, to hemolyze the red blood cells, the aliquots were diluted with water in same quantity at room temperature. After 2 minutes, the solution was centrifuged at $17000 \mathrm{~g}$ in $4^{\circ} \mathrm{C}$ for 15 second and the supernatant was separated. This centrifugation process was repeated to obtain a clear supernatant of the sample. To confirm the separation of erythrocyte membrane from leukocytes the sediment was transferred to a derivatization vial and the derivatization was performed directly on the washed erythrocyte cells.

Transesterification was completed by adding $2 \mathrm{~mL}$ of methanol:dichloromethane and $200 \mu \mathrm{L}$ of acetyl chloride to the red blood cell and incubating at $80^{\circ} \mathrm{C}$ for 60 minutes. To extract the fatty acid methyl esters (FAME), $2 \mathrm{~mL} n$-hexane was added to the solution and the n-hexane phase was washed with acetonitrile to purify FAME. The extract in n-hexane phase (FAME) was then dried with nitrogen stream and diluted with $1 \mathrm{~mL}$ dichloromethane. Subsequently, the presence of FAME were determined by Gas Chromatography (GC) equipped with Flame lonization Detector (FID) (Varian, CP-3800) and a capillary column (FFAP, 30 m, $0.22 \mathrm{~mm}$ film thickness, $0.25 \mathrm{~mm}$ i.d.), when helium was used as a carrier gas. The injection volume was $1 \mathrm{~mL}$ and the sample was injected in split-less mode. The temperature of the injector and detector (FID) were maintained at $255^{\circ} \mathrm{C}$ and $270^{\circ} \mathrm{C}$, respectively. The temperature of the column was programmed to reach to $125^{\circ} \mathrm{C}$ from $100^{\circ} \mathrm{C}$ (the primary oven temperature) with a hold for 0.5 minute, and then reached to $150^{\circ} \mathrm{C}$ at a rate of $25^{\circ} \mathrm{C} / \mathrm{min}$ with a hold time for 2 minutes and ultimately raised to $200^{\circ} \mathrm{C}$ at a rate of $25^{\circ} \mathrm{C} / \mathrm{min}$ with a hold for 37 minutes. Meanwhile the flow rate of carrier gas (helium), hydrogen and air were 25,30 and $300 \mathrm{ml} / \mathrm{min}$, respectively for the FID detector. The separated compounds were identified by comparing their retention times (RT), with the individual standards. The relative percent amount of each component was measured by comparing its average peak area to the total areas. All analyses were done in triplicate.

To quantify the fatty acids in the erythrocyte membrane, the areas of the peaks for lauric and docosahexanoic acid (DHA) were considered. A standard FAME mixture was used to calibrate the response of flame ionization detector (Supelco-37 FAME mix, Bellefont, PA, USA). To obtain total short chain fatty acid (SFA), the saturated fatty acids with carbon numbers 12:0; 14:0; 15:0; 16:0; 17:0; 18:0; 20:0; 22:0; 24:0 were considered. Total monounsatured fatty acids (MUFAs) were determined by summing 14:1n-5; 15:1n-5; 16:1n-7 + n-9; 17:1n-7; 18:1n-7 +n-9; 20:1n-9; 24:1n-9, and for n-6 polyunsatured fatty acids (PUFAs) the sum of $18: 2 n-6 ; 18: 3 n-6 ; 20: 2 n-6 ; 20: 3 n-6 ; 20: 4 n-6 ; 22: 2 n-6 ; 22: 4 n-6 ; 22: 5 n-6$ was used. Gradually the sum of $18: 3 n-3 ; 20: 3 n-3 ; 20: 5 n-3 ; 22: 5 n-3 ; 22: 6 n-3$ was used to calculate the total n-3 PUFA. The total of $18: 1$ and $18: 2$ trans isomers was considered as trans fatty acids. The sum of four erythrocyte-membrane measurements was calculated as erythrocyte - membrane total fatty acids.

\section{Measurement of macro- and micro-nutrient intake}

Apart from completing a FFQ by each participant, an interview was conducted by an expert dietitian. The portion size and weekly, monthly and annual frequencies of various dietary intakes was determined over last 12 months. The reproducibility and validity of 168 -items of FFQ were evaluated during the Tehran Lipid and Glucose Study. and similar approached was used in this study. The FFQ was analysed for macro- and micro-nutrient content by using NUTRITIONIST IIII software (version 7.0; N-Squared Computing, Salem, OR, USA), which was adapted for Iranian foods.

\section{Anthropometric measurements}

Body weight of the participants was measured in kilogram ( $\mathrm{kg}$ ) with lightweight clothing and no shoes by an accurate scale (Seca 813 Robusta, max $200 \mathrm{~kg}$, Hamburg, Germany). The height of individuals was also measured in centimetre (cm) by using a non-stretchable measuring tape and converted to meter before calculating body mass index (BMI). The BMI was calculated from the above-mentioned data using following formula: 
Body mass index $=(\mathrm{BMI}) \mathrm{kg} / \mathrm{m}^{2}$ [Body weight in $\mathrm{kg} /$ Square of the height in meter]

Waist circumference was determined by measuring waist diameter at the midpoint between the lower edge of the tenth rib cage and the iliac crests. The hip circumference was defined as the widest circumference measured at the hips and buttocks. Waist-to-hip ratio (WHR) was calculated from above-mentioned data.

\section{Physical activity}

Physical activity of military personnel was estimated by using an International Physical Activity Questionnaire adapted from Epic-Norfolk study, UK.

\section{Statistical analysis}

Recommended sample size for this study was 400 . This sample size led to a power of $80 \%$ and an a equal to 0.05 . The distribution of variables was studied with probability plots and the Shapiro-Wilks test. The relationship of the adiponectin and serum lipids level with dietary components was analyzed by Pearson's correlation coefficient. Partial correlation analyses were done with the adjustment for age, WHR, BMI and calorie intake. Multiple linear regression models were used to evaluate the relationship between dietary SFA, MUFA, PUFA, fiber intake and circulating adiponectin, serum lipids and FPG levels. A p value of $<0.05$ was considered as a significance of difference.

All statistical analyses were conducted with an IBM computer and the SPSS statistical software package (version 15, SPSS Inc., Chicago, IL, USA).

\section{Results}

A total of 400 personnel were recruited in the study. Their mean ( \pm SD) age was $34.67( \pm 6.61)$ years. Table 1 shows the basic characteristics of the study participants and their dietary intake of calorie, protein, carbohydrates, fiber, total fat, cholesterol, SFA, MUFA and PUFA. 
Table 1

Basic characteristics of the individuals participated in this study.

\begin{tabular}{|c|c|c|}
\hline Characteristics & Mean & SD \\
\hline Age (years) & 34.67 & 6.61 \\
\hline Body weight (kg) & 80.53 & 11.35 \\
\hline Height (cm) & 176.09 & 6.74 \\
\hline BMI $\left(\mathrm{kg} / \mathrm{m}^{2}\right)$ & 25.89 & 2.71 \\
\hline Waist circumference (cm) & 92.48 & 10.41 \\
\hline Hip circumference (cm) & 100.10 & 8.55 \\
\hline Waist - hip ratio (WHR) & 0.92 & 0.04 \\
\hline \multicolumn{3}{|l|}{ Education } \\
\hline Diploma & $7(2.5 \%)$ & \\
\hline Associate degree & $106(35 \%)$ & \\
\hline Bachelor degree & $163(45 \%)$ & \\
\hline Master degree & $23(7 \%)$ & \\
\hline Cigarette smoker & 8 & \\
\hline Non-smoker & 292 & \\
\hline \multicolumn{3}{|l|}{ Marital status } \\
\hline Single & $36(12 \%)$ & \\
\hline Married & $264(88 \%)$ & \\
\hline \multicolumn{3}{|c|}{ Serum parameters and blood pressure } \\
\hline FBS (mg/dL) & 90.55 & 16.95 \\
\hline TG (mg/dL) & 141.67 & 52.26 \\
\hline Cholesterol (mg/dL) & 165.17 & 33.99 \\
\hline LDL-c (mg/dL) & 98.16 & 21.00 \\
\hline $\mathrm{HDL}-\mathrm{C}(\mathrm{mg} / \mathrm{dL})$ & 38.84 & 7.31 \\
\hline Systolic BP & 119.74 & 10.09 \\
\hline Diastolic BP & 79.43 & 7.20 \\
\hline Adiponectin $(\mu \mathrm{g} / \mathrm{mL})$ & 12.38 & 9.54 \\
\hline \multicolumn{3}{|l|}{ Physical activity level } \\
\hline Inactive & $11.3 \%$ & \\
\hline Moderately inactive & $24.9 \%$ & \\
\hline Moderately active & $32.4 \%$ & \\
\hline Active & $26.5 \%$ & \\
\hline \multicolumn{3}{|l|}{ Dietary intake } \\
\hline Calorie intake (kcal/d) & 2258.48 & 790.83 \\
\hline Carbohydrate intake (g/d) & 377.44 & 143.88 \\
\hline Total fat intake ( $\mathrm{g} / \mathrm{d})$ & 55.72 & 53.56 \\
\hline Protein intake ( $\mathrm{g} / \mathrm{d}$ ) & 82.71 & 34.23 \\
\hline SFA intake (g/d) & 12.75 & 14.30 \\
\hline MUFA intake (g/d) & 7.49 & 11.82 \\
\hline PUFA intake (g/d) & 4.92 & 10.84 \\
\hline
\end{tabular}

Data are presented as mean \pm SD $(n=400)$. BMI, body mass index; FBS, Fasting blood sugar; HDL-c, High density lipoprotein cholesterol; MUFA, LDL-C, Low density lipoprotein cholesterol; monounsaturated fatty acid; PUFA, polyunsaturated fatty acid; SFA, saturated fatty acid; TG, Triglyceride; SD, standard deviation; WHR, waist-hip ratio. 


\begin{tabular}{|ll|}
\hline Characteristics & Mean \\
\hline Fiber intake $(\mathrm{g} / \mathrm{d})$ & 7.88 \\
\hline Cholesterol intake $(\mathrm{mg} / \mathrm{d})$ & 515.20 \\
\hline $\begin{array}{l}\text { Data are presented as mean } \pm \text { SD }(\mathrm{n}=400) \text {. BMI, body mass index; FBS, Fasting blood sugar; HDL-c, High density lipoprotein cholesterol; MUFA, LDL-c, Low } \\
\text { density lipoprotein cholesterol; monounsaturated fatty acid; PUFA, polyunsaturated fatty acid; SFA, saturated fatty acid; TG, Triglyceride; SD, standard } \\
\text { deviation; WHR, waist-hip ratio. }\end{array}$ \\
\hline
\end{tabular}

The results showed that the prevalence of diabetes mellitus and pre-diabetes were $3.3 \%(n=13)$ and $13.3 \%(n=53)$, respectively. The prevalence of hypercholestrplemia was $13 \%(n=52)$ and hypertriglyceridemia was $30.4 \%(n=122)$. The prevalence of overweight and obesity were $13.7 \%(n=55)$ and $4.7 \%$ $(n=19)$, respectively. Waist circumference more than $100 \mathrm{~cm}$ was considered as obesity and it was $14.4 \%(n=58)$. A 10.4\% $(n=42)$ of participated military personnel had systolic hypertension and $14.7 \%(n=59)$ had diastolic hypertension.

The average calorie intake through carbohydrate, fat and protein intake of the total calorie consumption was $67 \%, 22 \%$ and $11 \%$, respectively. The mean percent calorie intake from dietary SFA was $5 \pm 6 \%$ of total calorie intake.

\section{The adjusted and un-adjusted correlations between various erythrocyte membrane fatty acids and serum adiponectin and lipids}

The Pearson correlation showed a significant inverse relationship between total SFA, Lauric, Myristic and Palmitic acid measured in the erythrocyte membrane and serum adiponectin level before and after adjustment for age, weight, BMI, WHR and calorie intake (Table 2). In univariate analysis, there was also negative significant association between total SFA $(\beta=-0.243, p=0.023)$, Lauric $(\beta=-0.204, p=0.012)$, Myristic $(\beta=-0.122, p=0.029)$ and Palmitic acid $(\beta=-0.172, p=$ 0.033 ) in erythrocyte-membrane and serum adiponectin level (Table 3$)$.

Table 2

Age, BMI, WHR and calorie adjusted and unadjusted correlations between various fatty acids measured in erythrocyte membrane :

\begin{tabular}{|c|c|c|c|c|c|c|c|c|c|c|c|c|c|c|}
\hline & \multicolumn{4}{|c|}{ Adiponectin } & \multicolumn{4}{|c|}{ Triglycerides } & \multicolumn{4}{|c|}{ Cholesterol } & \multicolumn{2}{|c|}{ LDL-cholesterol } \\
\hline & $\begin{array}{l}\text { Un- } \\
\text { adjusted }\end{array}$ & & Adjusted & & $\begin{array}{l}\text { Un- } \\
\text { adjusted }\end{array}$ & & Adjusted & & $\begin{array}{l}\text { Un- } \\
\text { adjusted }\end{array}$ & & Adjusted & & $\begin{array}{l}\text { Un- } \\
\text { adjustec }\end{array}$ & \\
\hline & $r$ & $\mathrm{p}$ & $r$ & $\mathrm{p}$ & $r$ & $p$ & $r$ & $\mathrm{p}$ & $r$ & $\mathrm{p}$ & $r$ & $\mathrm{p}$ & $r$ & $\mathrm{p}$ \\
\hline SFA & -0.21 & 0.001 & -0.20 & 0.01 & 0.08 & 0.43 & 0.05 & 0.47 & 0.27 & 0.001 & 0.25 & 0.001 & 0.19 & 0.03 \\
\hline $\begin{array}{l}\text { Lauric acid } \\
(12: 0)\end{array}$ & -0.25 & 0.02 & -0.18 & 0.03 & 0.002 & 0.64 & 0.001 & 0.58 & 0.23 & 0.01 & 0.20 & 0.01 & 0.22 & 0.02 \\
\hline $\begin{array}{l}\text { Myristic } \\
\text { acid (14:0) }\end{array}$ & -0.22 & 0.001 & -0.20 & 0.01 & 0.005 & 0.78 & 0.002 & 0.39 & 0.19 & 0.001 & 0.18 & 0.01 & 0.23 & 0.01 \\
\hline $\begin{array}{l}\text { Palmitic } \\
\text { acid (16:0) }\end{array}$ & -0.27 & 0.001 & -0.22 & 0.02 & 0.04 & 0.29 & 0.005 & 0.88 & 0.17 & 0.02 & 0.15 & 0.02 & 0.22 & 0.001 \\
\hline $\begin{array}{l}\text { Stearic acid } \\
(18: 0)\end{array}$ & 0.03 & 0.68 & 0.006 & 0.42 & 0.006 & 0.70 & 0.001 & 0.69 & .0 .005 & 0.24 & 0.002 & 0.63 & 0.02 & 0.51 \\
\hline MUFA & 0.18 & 0.04 & 0.15 & 0.04 & 0.005 & 0.53 & 0.004 & 0.35 & -0.18 & 0.03 & -0.15 & 0.04 & -0.20 & 0.002 \\
\hline PUFA & -0.05 & 0.44 & -0.01 & 0.83 & 0.006 & 0.83 & 0.001 & 0.56 & 0.02 & 0.34 & 0.01 & 0.23 & 0.005 & 0.71 \\
\hline$n-3$ & 0.15 & 0.03 & 0.14 & 0.02 & 0.004 & 0.34 & 0.005 & 0.46 & 0.07 & 0.87 & 0.009 & 0.32 & 0.002 & 0.58 \\
\hline $\begin{array}{l}\text { a-Linolenic } \\
\text { acid }(18: 3 n- \\
3)\end{array}$ & 0.03 & 0.75 & 0.01 & 0.41 & 0.01 & 0.57 & 0.04 & 0.57 & 0.01 & 0.12 & 0.009 & 0.78 & 0.03 & 0.62 \\
\hline $\begin{array}{l}\text { EPA }(20: 5 n- \\
3)\end{array}$ & 0.24 & 0.001 & 0.19 & 0.01 & 0.07 & 0.45 & 0.04 & 0.35 & .0 .001 & 0.76 & 0.001 & 0.59 & 0.002 & 0.35 \\
\hline $\begin{array}{l}\text { DHA } \\
(22: 6 n-3)\end{array}$ & 0.12 & 0.04 & 0.11 & 0.03 & 0.004 & 0.62 & 0.003 & 0.88 & .0 .01 & 0.67 & 0.001 & 0.32 & 0.07 & 089 \\
\hline$n-6$ & 0.003 & 0.75 & 0.001 & 0.85 & 0.007 & 0.35 & 0.001 & 069 & 0.07 & 0.87 & 0.01 & 0.44 & 0.02 & 088 \\
\hline $\begin{array}{l}\text { Linoleic } \\
\text { acid }(18: 2 n- \\
6)\end{array}$ & 0.007 & 0.65 & 0.002 & 0.84 & 0.04 & 0.31 & 0.03 & 0.43 & 0.22 & 0.04 & 0.15 & 0.03 & 0.15 & 0.03 \\
\hline $\begin{array}{l}\text { Arachidonic } \\
\text { acid }(20: 4 n- \\
6)\end{array}$ & 0.04 & 0.74 & 0.01 & 0.58 & 0.001 & 0.89 & 0.001 & 0.38 & 0.02 & 0.32 & 0.01 & 0.87 & 0.03 & 0.44 \\
\hline $\begin{array}{l}\text { Trans Fatty } \\
\text { Acids }\end{array}$ & -0.06 & 0.38 & -0.02 & 0.65 & 0.05 & 0.71 & 0.03 & 0.51 & 0.28 & 0.001 & 0.30 & 0.001 & 0.29 & 0.001 \\
\hline
\end{tabular}

BMI, Body mass index; DHA, Docosahexanoic acid; EPA, Eicosapentanoic acid; HDL-C, High density lipoprotein cholesterol; LDL-C, Low density lipoprotein che Polyunsaturated fatty acids; SFA, Saturated fatty acids; WHR, Waist hip ratio. 
Table 3

Multiple linear regression analysis for the association between fatty acids measured in erythrocyte membrane and serum adiponectin, triglycerides, cholesterol, LDL-C and HDL-c in army personnel.

\begin{tabular}{|c|c|c|c|c|c|c|c|c|c|c|}
\hline & \multicolumn{2}{|c|}{ Adiponectin } & \multicolumn{2}{|c|}{ Triglycerides } & \multicolumn{2}{|c|}{ Cholesterol } & \multicolumn{2}{|c|}{ LDL-cholesterol } & \multicolumn{2}{|c|}{ HDL-cholesterol } \\
\hline & $\beta$ & $\mathrm{p}$ & $\beta$ & $\mathrm{p}$ & $\beta$ & $\mathrm{p}$ & $\beta$ & $\mathrm{p}$ & $\beta$ & $\mathrm{p}$ \\
\hline Total SFA & -0.243 & 0.023 & 0.012 & 0.409 & 0.210 & 0.002 & 0.197 & 0.026 & 0.013 & 0.783 \\
\hline Lauric acid (12:0) & -0.204 & 0.012 & 0.021 & 0.908 & 0.182 & 0.034 & 0.207 & 0.001 & 0.026 & 0.623 \\
\hline Myristic acid (14:0) & -0.122 & 0.029 & 0.003 & 0.476 & 0.127 & 0.048 & 0.155 & 0.025 & 0.003 & 0.325 \\
\hline Palmitic acid (16:0) & -0.172 & 0.033 & 0.009 & 0.971 & 0.188 & 0.038 & 0.195 & 0.029 & 0.007 & 0.599 \\
\hline Stearic acid (18:0) & 0.038 & 0.459 & 0.044 & 0.612 & -0.009 & 0.471 & -0.004 & 0.612 & 0.005 & 0.633 \\
\hline MUFA & 0.017 & 0.236 & -0.006 & 0.322 & -0.173 & 0.046 & -0.199 & 0.022 & 0.023 & 0.722 \\
\hline PUFA & 0.244 & 0.021 & -0.030 & 0.774 & -0.201 & 0.017 & -0.184 & 0.018 & 0.006 & 0.341 \\
\hline$n-3$ & 0.226 & 0.009 & -0.014 & 0.436 & -0.024 & 0.577 & -0.001 & 0.744 & 0.012 & 0.977 \\
\hline a-Linolenic acid (18:3n-3) & -0.011 & 0.977 & -0.028 & 0.497 & -0.001 & 0.355 & -0.025 & 0.097 & 0.042 & 0.325 \\
\hline EPA (20:5n-3) & 0.156 & 0.026 & -0.007 & 0.644 & -0.027 & 0.892 & -0.019 & 0.582 & 0.006 & 0.994 \\
\hline DHA (22:6n-3) & 0.220 & 0.042 & -0.038 & 0.591 & -0.026 & 0.845 & -0.009 & 0.855 & 0.019 & 0.341 \\
\hline$n-6$ & 0.031 & 0.548 & 0.038 & 0.482 & -0.184 & 0.031 & -0.199 & 0.037 & 0.002 & 0.114 \\
\hline Linoleic acid (18:2n-6) & 0.002 & 0.576 & 0.001 & 0.645 & -0.216 & 0.017 & -0.207 & 0.021 & 0.005 & 0.833 \\
\hline Arachidonic acid (20:4n-6) & 0.025 & 0.899 & 0.013 & 0.986 & 0.012 & 0.241 & 0.028 & 0.099 & 0.016 & 0.839 \\
\hline Trans fatty acids & 0.011 & 0.387 & 0.015 & 0.631 & 0.265 & 0.048 & 0.196 & 0.045 & 0.001 & 0.966 \\
\hline
\end{tabular}

Also a positive significant correlation was found between total n-3 PUFA, EPA and DHA derived from erythrocyte membrane with serum adiponectin level before and after adjustment for age, weight, BMI, WHR and calorie intake (Table 2). Moreover, in univariate regression analysis we found a positive association between $n-3$ PUFA $(\beta=0.226, p=0.009)$, EPA $(\beta=-0.156, p=0.026)$ and DHA $(\beta=0.220, p=0.042)$ derived from erythrocyte-membrane and adiponectin after adjustment for age, weight, BMI, WHR and calorie intake (Table 3).

Bivariate correlation analysis depicted a significant positive correlation between total SFA, Lauric, Myristic and Palmitic acid measured in the erythrocyte membrane with serum total cholesterol and LDL-c level before and after adjustment for age, weight, BMI, WHR and calorie intake (Table 2). This significant relationship persisted in the univariate regression analyses for total SFA $(\beta=0.210, p=0.002)$, Lauric $(\beta=0.182, p=0.034)$, Myristic $(\beta=0.127, p=0.048)$ and Palmitic acid $(\beta=0.188, p=0.038)$ measured in the erythrocyte membrane and serum total cholesterol after adjustment for age, BMI, WHR and total calorie intake (Table 3). Moreover, in univariate regression analysis, a positive association was observed between total SFA $(\beta=0.197, p=0.026)$, Lauric ( $\beta=0.207, p$ $=0.001)$, Myristic $(\beta=0.155, p=0.025)$ and Palmitic acid $(\beta=0.195, p=0.029)$ measured in the erythrocyte membrane and serum LDL-c after the adjustment for age, BMI, WHR and total calorie intake (Table 3).

Based on the obtained data a negative significant association was found between Linoleic acid measured in erythrocyte membrane with serum total cholesterol and LDL-c levels (Table 2). In the multiple regression analyses, Linoleic acid measured in erythrocyte membrane showed a significant negative correlation with serum cholesterol level $(\beta=-0.216, p=0.017)$, and LDL-c $(\beta=-0.207, p=0.021)$ after controlling for age, $B M I, W H R$ and total calorie intake (Table 3).

There was no significant correlation between trans fatty acids in erythrocyte membrane and adiponectin and other serum lipids.

\section{The dietary fatty acid intake and adiponectin and serum lipid profile}

There was a significant inverse but weak correlation between dietary SFA intake and serum adiponectin level was observed, which persisted even after the adjustment for age, BMI, WHR and total calorie intake (Table 4). Multiple regression analyses showed that, dietary SFA intake had a significant negative and weak correlation with serum adiponectin level $(\beta=-0.079, p=0.034)$ after the adjustment for age, $B M I$, WHR and total calorie intake (Table 5). 
Table 4

Age, BIM, WHR, calorie intake adjusted and un-adjusted correlation between dietary MUFA, PUFA, SFA, cholesterol, carbohydrate, fiber aı

\begin{tabular}{|c|c|c|c|c|c|c|c|c|c|c|c|c|c|c|c|}
\hline & \multicolumn{4}{|c|}{ Adiponectin } & \multicolumn{4}{|c|}{ Triglycerides } & \multicolumn{4}{|c|}{ Cholesterol } & \multicolumn{3}{|c|}{ LDL-cholesterol } \\
\hline & \multicolumn{2}{|l|}{$\begin{array}{l}\text { Un- } \\
\text { adjusted }\end{array}$} & \multicolumn{2}{|l|}{ Adjusted } & \multicolumn{2}{|c|}{$\begin{array}{l}\text { Un- } \\
\text { adjusted }\end{array}$} & \multicolumn{2}{|l|}{ Adjusted } & \multicolumn{2}{|c|}{$\begin{array}{l}\text { Un- } \\
\text { adjusted }\end{array}$} & \multicolumn{2}{|l|}{ Adjusted } & \multicolumn{2}{|c|}{$\begin{array}{l}\text { Un- } \\
\text { adjusted }\end{array}$} & \multirow{2}{*}{$\begin{array}{l}\mathrm{Ad} \\
\mathrm{r}\end{array}$} \\
\hline & $r$ & $\mathrm{p}$ & $r$ & $\mathrm{p}$ & $r$ & $\mathrm{p}$ & $r$ & $\mathrm{p}$ & $r$ & $p$ & $r$ & $\mathrm{p}$ & $r$ & $p$ & \\
\hline $\begin{array}{l}\text { Dietary } \\
\text { fat }\end{array}$ & -0.03 & 0.49 & -0.002 & 0.82 & -0.06 & 0.59 & -0.001 & 0.89 & 0.09 & 0.42 & 0.008 & 0.38 & -0.06 & 0.58 & 0.0 \\
\hline $\begin{array}{l}\text { Dietary } \\
\text { SFA }\end{array}$ & -0.10 & 0.001 & -0.09 & 0.01 & -0.07 & 0.56 & -0.08 & 0.43 & 0.12 & 0.01 & 0.09 & 0.03 & 0.12 & 0.02 & 0.1 \\
\hline $\begin{array}{l}\text { Dietary } \\
\text { MUFA }\end{array}$ & -0.02 & 0.51 & -0.01 & 0.87 & 0.02 & 0.44 & 0.03 & 0.71 & -.0 .09 & 0.02 & -0.08 & 0.04 & -0.10 & 0.04 & -0 \\
\hline $\begin{array}{l}\text { Oleic } \\
\text { acid }\end{array}$ & -0.07 & 0.82 & -0.05 & 0.23 & 0.08 & 0.39 & 0.09 & 0.69 & -.0 .12 & 0.01 & -0.10 & 0.02 & -0.13 & 0.01 & -0 \\
\hline $\begin{array}{l}\text { Dietary } \\
\text { PUFA }\end{array}$ & -0.002 & 0.22 & -0.001 & 0.49 & 0.05 & 0.32 & 0.001 & 0.67 & -0.09 & 0.02 & -0.14 & 0.03 & -0.13 & 0.03 & -0.1 \\
\hline $\begin{array}{l}\text { Linoleic } \\
\text { acid }\end{array}$ & -0.005 & 0.78 & -0.008 & 0.82 & 0.02 & 0.76 & 0.008 & 0.35 & -0.10 & 0.01 & -0.12 & 0.02 & -0.10 & 0.04 & -0. \\
\hline $\begin{array}{l}\text { Linolenic } \\
\text { acid }\end{array}$ & -0.009 & 0.72 & -0.006 & 0.35 & 0.009 & 0.45 & 0.005 & 0.73 & -0.12 & 0.03 & -0.11 & 0.04 & -0.09 & 0.01 & -0. \\
\hline $\begin{array}{l}\text { n-3 fatty } \\
\text { acids }\end{array}$ & -0.007 & 0.45 & -0.003 & 0.76 & 0.01 & 040 & 0.002 & 0.72 & 0.03 & 0.97 & 0.005 & 0.81 & 0.006 & 0.58 & 0.0 \\
\hline $\begin{array}{l}\text { Dietary } \\
\text { carb. }\end{array}$ & -0.001 & 0.23 & -0.01 & $\begin{array}{l}0.5 \\
2\end{array}$ & 0.08 & 0.01 & 0.06 & 0.01 & 0.21 & 0.47 & 0.19 & 0.45 & 0.19 & 0.68 & 0.1 \\
\hline $\begin{array}{l}\text { Dietary } \\
\text { chol. }\end{array}$ & 0.001 & 0.84 & .0001 & 0.80 & 0.034 & 048 & 0.078 & 0.99 & .001 & 0.98 & .0001 & 0.99 & 0.094 & 0.37 & .00 \\
\hline $\begin{array}{l}\text { Dietary } \\
\text { fiber }\end{array}$ & -0.07 & 0.31 & -0.04 & 0.48 & 0.08 & 0.50 & 0.09 & 0.83 & 0.24 & 0.03 & 0.09 & 0.04 & 0.20 & 0.01 & 0.1 \\
\hline
\end{tabular}

Table 5

Multiple linear regression analysis for the association between dietary lipids and serum adiponectin, triglycerides, cholesterol, LDL-c and HDL-c in army

\begin{tabular}{|c|c|c|c|c|c|c|c|c|c|c|}
\hline & \multicolumn{2}{|c|}{ Adiponectin } & \multicolumn{2}{|c|}{ Triglycerides } & \multicolumn{2}{|c|}{ Cholesterol } & \multicolumn{2}{|c|}{ LDL-cholesterol } & \multicolumn{2}{|c|}{ HDL-cholesterol } \\
\hline & $\beta$ & $\mathrm{p}$ & $\beta$ & $\mathrm{p}$ & $\beta$ & $\mathrm{p}$ & $\beta$ & $\mathrm{p}$ & $\beta$ & $p$ \\
\hline Dietary SFA & -0.079 & 0.034 & 0.017 & 0.565 & 0.122 & 0.011 & 0.135 & 0.001 & 0.004 & 0.257 \\
\hline Dietary PUFA & 0.013 & 0.728 & -0.014 & 0.237 & -0.099 & 0.034 & -0.104 & 0.022 & 0.072 & 0.542 \\
\hline Linoleic acid & 0.025 & 0.651 & -0.073 & 0.755 & -0.138 & 0.039 & -0.056 & 0.042 & 0.044 & 0.673 \\
\hline Linolenic acid & 0.078 & 0.343 & -0.094 & 0.459 & -0.096 & 0.065 & -0.109 & 0.055 & 0.019 & 0.744 \\
\hline n-3 fatty acids & 0.064 & 0.046 & -0.075 & 0.877 & -0.036 & 0.445 & -0.011 & 0.726 & 0.099 & 0.457 \\
\hline Dietary MUFA & 0.039 & 0.931 & -0.016 & 0.233 & -0.161 & 0.001 & -0.102 & 0.001 & 0.001 & 0.733 \\
\hline Oleic acid & 0.092 & 0.787 & -0.025 & 0.582 & -0.119 & 0.012 & -0.096 & 0.024 & 0.045 & 0.564 \\
\hline Dietary cholesterol & 0.021 & 0.355 & 0.0001 & 0.359 & 0.043 & 0.121 & 0.087 & 0.557 & 0.054 & 0.743 \\
\hline Dietary carbohydrate & 0.008 & 0.793 & 0.0003 & 0.798 & 0.026 & 0.101 & 0.019 & 0.349 & 0.032 & 0.378 \\
\hline Dietary fiber & 0.027 & 0.872 & 0.001 & 0.347 & 0.018 & 0.093 & 0.046 & 0.787 & 0.024 & 0.741 \\
\hline
\end{tabular}

No significant correlation was found between dietary PUFA, MUFA and cholesterol intake and serum adiponectin level (Table 4).

Bivariate correlation analysis showed a significant positive correlation between dietary SFA intake and serum cholesterol and LDL-c level. This significant relationship persisted after adjustment for age, BMI, WHR and total calorie intake (Table 4). In addition, a negative significant association was found between 
dietary PUFA and MUFA with serum total cholesterol and LDL-c level (Table 4). In the multiple regression analyses, dietary SFA intake showed a significant positive correlation with serum cholesterol level $(\beta=0.122, p=0.011)$, and LDL-c $(\beta=0.135, p=0.001)$ after controlling for age, $B M I$, WHR and total calorie intake (Table 5). In addition, dietary PUFA intake revealed a significant inverse association with serum cholesterol level ( $\beta=-0.099, p=0.034)$, and LDL-C $(\beta=-0.104, p=0.022)$ following adjustment for age, BMI, WHR and total calorie intake (Table 5). A significant negative correlation was also found between dietary MUFA intake and serum cholesterol level $(\beta=-0.161, p=0.001)$, and LDL-C $(\beta=-0.102, p=0.001)$ after the adjustment for age, BMI, WHR and total calorie intake (Table 5).

Furthermore, dietary Linoleic acid intake revealed a significant negative association with serum cholesterol level $(\beta=-0.138, p=0.039)$, and LDL-c $(\beta=-0.056, p$ $=0.042$ ) following adjustment for age, BMI, WHR and total calorie intake (Table 5).

Bivariate correlation analysis showed a significant reveres correlation between dietary fiber intake and serum cholesterol and LDL-c level. This significant relationship persisted after adjustment for age, BMI, WHR and total calorie intake (Table 4).

No significant association was seen between dietary SFA, PUFA and MUFA with serum HDL-c (Tables 4 and 5). There was no correlation between dietary SFA, PUFA and MUFA with serum TG level. However, a significant direct association was found between dietary carbohydrate and serum TG level. This relationship persists after controlling for age, WHR, total energy intake and BMI (Tables 4 and 5).

\section{Discussion}

Present study was conducted to examine the correlations between dietary fatty acid intake with fatty acid compositions of erythrocyte cell membrane, serum adiponectin and lipid levels. Based on our findings there was an inverse and significant relationship between total SFA including lauric, stearic and palmitic acid of the erythrocyte membrane with serum adiponectin level in army population. Some literatures have reported that consumption of SFA suppress the production of adiponectin in humans and rodents [21]. In addition, Fernandez-Real et al. [22] demonstrated a negative correlation between serum palmitate concentration and circulating adiponectin level in non-army individuals [22]. Some reports indicated that high serum concentration of SFA level usually increases due to obesity and the negative impact of obesity on adiponectin might explain the effect of serum SFA on adiponectin [23]. Adiponectin is an antiinflammatory cytokine and its suppression or expression depends on the other mediators. It has been reported that tumor necrosis factor- alpha (TNF- $\mathrm{a}$ ) and nuclear factor kappa-B (NF-KB) suppresses the production of adiponectin and interlukin-6 (IL-6) and peroxisome proliferators activated receptors $y$ (PPAR- $\gamma$ ) activate adiponectin expression [24]. The inhibitory effect of SFA on PPAR- $\gamma$ is mediated by activating TNF-alpha synthesis and this process might elucidate the effect of dietary and erythrocyte - membrane SFA on adiponectin level. Bradley et al; [25] demonstrated that serum palmitic acid activates the expression of mRNAs for TNF- alpha and NF-KB, [25] which in turn suppressed PPARy expression to decrease the adiponectin production [24].

In our study, no significant association was found between the dietary intake of n-6 PUFA and MUFA such as linoleic acid, linolenic and oleic acid with serum adiponectin level. However, a direct significant relationship was observed between linoleic acid measured in erythrocyte membrane and serum adiponectin concentration. Enzenbach and colleagues. [26] also reported a direct significant correlation between erythrocyte-membrane linoleic acid and adiponectin level [26]. The activation of PPAR-y by linoleic acid derivatives might be responsible for this result [27]. Earlier studies have shown that linoleic acid participates in anti-inflammatory signalling pathways [28] and these property might responsible for the direct relationship between linoleic acids derived from erythrocytemembrane with adiponectin concentration .

The findings of current study depicted a significant positive correlation between erythrocyt-mambrane n-3 fatty acids with adiponectin level in army population. The ability of n-3 fatty acids to increase adiponectin level is attributed to bind and activate of PPAR-y [27].

Overall, stronger significant association was observed between the fatty acids measured in erythrocyte membrane with adiponectin compared to those obtained from FFQ. Some previous studies [29] could not find a significant correlation between dietary fatty acids, determined by dietary questionnaire, and adipokines. It should be noted that the content of fatty acids in tissues or erythrocyte affects by uptake in different tissues, metabolism, excretion and genetics [30]. Therefore, it is hard to predict the pattern of the fatty acids in tissues from dietary sources. On the other hand, due to approximately 120 days life span, erythrocytes membrane fatty acid composition largely relies on the dietary pattern of preceding 120 days [31].

However, the outcome of current study showed a positive significant association between dietary SFA intake and total SFA derived from erythrocyte-membrane and serum LDL-c while a negative association between dietary PUFA and MUFA intake and serum LDL-c level. The 4 families of transcription factors: the peroxisome proliferators activated receptors (PPARs), liver X receptors (LXRs), hepatic nuclear factor-4 (HNF-4), and sterol regulatory element binding proteins (SREBPs) determine the roles of different fatty acids such as SFA, PUFA and MUFA on serum lipid profile [32]. SFA enhance the transcription of lipogenic genes by activating SREBP-1, [33] while the role of SREBPs in metabolism of cholesterol is proven and could be found elsewhere [34]. Furthermore, SFA raise serum LDL-c levels by decreasing LDL-c receptors, [35] while, PUFA decreases serum cholesterol and LDL-c level by inducing the fluidity of hepatocyte membrane which in turn increases LDL receptors [36]. In addition, PUFA increases LDL catabolism and lower LDL apo-B protein pool size, [37] and ultimately induces LXRa expression in liver [38] and suppresses SREBP-1 gene transcription LXR regulates intracellular cholesterol levels by inducing the expression of cholesterol 7a-hydroxylase (CYP7) in the process of bile acids synthesis [34] and SREBP-1 is responsible for lipogenesis and metabolism of cholesterol in liver [34]. In current study, a negative correlation was found between dietary MUFA intake with serum total cholesterol and LDL-c, which is in agreement with the results of several previous studies, which found a reduction in total cholesterol, [39], LDL-c, [40] and increases in HDL-c [41] following the consumption of a rich MUFA diet. The mechanism which underlies the hypocholestrolemic effect of MUFA might be attributed to the promotion of hepatic stearoyl-CoA desaturase-1 (SCD1) and suppressing SREBP activity [42].

In this study, the serum TG level of participants was significantly and positively correlated with dietary carbohydrate intake. The recent studies also imply an increase in TG level sequel to increasing carbohydrate consumption [43]. The effect of dietary carbohydrate intake on raising insulin level might explain this

Page $9 / 13$ 
phenomenon. One explanation is that higher insulin level could influence the liver to delay chylomicron elimination by corresponding receptors which in turn leads to hypertriglyceridemia [44]. Based on the result of our study, although dietary carbohydrate intake did not correlate with serum total cholesterol or LDL-c level, some studies indicated that rich carbohydrate diet leads to create small dense LDL-c in circulation which are more atherogenic than light larger LDL-C [45]. However, we didn't measure the size of the particles of LDL and it was one of the limitations of this study.

On the other hand, total dietary fiber intake in this study was inversely associated with serum LDL-c level (Table 4). The results published by Trustwell et al. [46] are in agreement with our finding that reported a potentially desire effect of dietary fiber on lipids and lipoproteins, [46] while reducing bile acid absorption is the key explanation for this effect [47]. In another study, Kelly and et al. [48] reported that the average reduction in total cholesterol when compared between high and low fiber diets was $0.17 \mathrm{mmol} / \mathrm{l}[48]$.

In general, the data of this study showed that in army population, the risk factors of CVD were lower than risk factors in civilian community in Iran. In third national health survey, the prevalence of risk factor for CVD in civilian community were $8.7 \%$ with diabetes, $9.2 \%$ with IFG, $36.4 \%$ with hypertriglyceridemia, $42.9 \%$ with hypercholestridemia, $26.6 \%$ with hypertension, $22.3 \%$ with obesity, and $53.6 \%$ with central obesity, [49] whereas for army personnel these parameters were $3.3 \%$ with diabetes, $13.3 \%$ with IGF, $30.4 \%$ with hypertriglyceridemia, $13 \%$ with hypercholestridemia, $24 \%$ with hypertension, $4.7 \%$ with obesity and $14.4 \%$ with central obesity, respectively. Cloeren and Mallon. [50] reported that the lower prevalence of CVD risk factors in army force is attributed to their desirable body shape compared to civilian population [50]. In current study, the prevalence of central obesity in army population was significantly lower than central obesity in general population.

\section{Limitation and strength}

The potential limitation of this study was that it was a cross sectional study which does not prove the causal relationships. However, investigating the relations between variables can propose mechanisms which may be involved in intervention studies. Another limitation was that we used NUTRITIONIST IIII software that was not able to analyze n-3 fatty acid content separately (DHA, EPA and a-Linolenic acid); therefore it was impossible to assess the associations between DHA, EPA, a-Linolenic acid and adiponectin level.However, the data for nutrient intake were taken from food FFQ and content of erythrocyte membrane which were based on the classification of dietary standards for epidemiological studies which can be considered as a potential strength of this study.

\section{Conclusion}

Based on the findings of this study, it is concluded that certain dietary nutrients might be associated with lower serum adiponectin levels, and suggest that inflammatory responses might be suppressed, at least in part, by dietary modifications. In addition, we found that dietary SFA content leads to elevate serum cholesterol and to reduce serum adiponectin levels in army personnel. It was also found a significant relationship between dietary carbohydrate intake and serum TG level and this might represent an important causal factor in the development of cardiovascular disease which potentially endangers the health of military personnel.

\section{Abbreviations}

CVD Cardiovascular disease

TG Triglyceride

LDL-c LDL-cholesterol

HDL-c HDL-cholesterol

MUFA Monounsaturated fatty acids

PUFA Polyunsaturated fatty acids

n-3 PUFAs n-3 polyunsaturated fatty acids

FFQ Food frequency questionnaire

FPG Fasting plasma glucose

VLDL-c Very low density lipoprotein-cholesterol

FAME Fatty acid methyl esters

GC Gas Chromatography

FID Flame lonization Detector

RT Retention time

DHA Docosahexanoic acid 
BMI Body mass index

WHR Waist-to-hip ratio

TNF-a Tumor necrosis factor- alpha

NF-Kb Nuclear factor kappa-B

IL-6 Interlukin-6

PPAR-y Peroxisome proliferators activated receptors $y$

LXRs Liver $\mathrm{X}$ receptors

HNF-4 Hepatic nuclear factor-4

SREBPs Sterol regulatory element binding proteins

SCD1 Stearoyl-CoA desaturase-1

\section{Declarations}

\section{Ethics approval and consent to participate}

The research protocol was approved by ethic committee of Baqiyatallah University of Medical Sciences (IR.BMSU.REC.1398.056), Tehran, Iran.

\section{Consent for publication}

An informed consent form was completed by each participating individual.

\section{Availability of data and materials}

The datasets used and/or analyzed during the current study are available from the corresponding author on reasonable request.

\section{Competing interests}

The authors declare that they have no competing interests.

\section{Funding}

This work was financially supported by The Baqiyatallah University of Medical Sciences, Tehran, I.R. Iran. [Grant Number: 97000548].

\section{Authors' contributions}

H. R: Conception and design of the study, revising the article critically for important intellectual content, Acquisition of data, M. S: Acquisition of data, drafting the article, M. S: Analysis and interpretation of the data.

\section{Acknowledgements}

Not applicable

\section{References}

1. Ramsey CB, Hostetler C, Andrews A. Evaluating the nutrition intake of U.S. military service members in garrison. Mi Med. 2013;178:1285-90.

2. Barringer N, Pasiakos S, McClung H, Crombie A, Margolis L. Prediction equation for estimating total daily energy requirements of special operations personnel. J Int Soc Sports Nutr. 2018;15:15.

3. Doughty H, Woolley T, Thomas G. Massive transfusion. Army Med Corps. 2011;157 Suppl 3:277-S83.

4. Margolis LM, Rood J, Champagne C, Young AJ, Castellani JW. Energy balance and body composition during US Army special forces training. Appl Physiol Nutr Metab. 2013;38:396-400.

5. Crombie AP, Funderburk LK, Smith TJ, McGraw SM, Walker LA, Champagne CM, et al. Effects of modified foodservice practices in military dining facilities on ad libitum nutritional intake of US army soldiers. J Acad Nutr Diet. 2013;113:920-7.

6. Lacroix S, Cantin J, Nigam A. Contemporary issues regarding nutrition in cardiovascular rehabilitation. Phys Rehabil Med. 2017;60:36-42.

7. Bingham CM, Lahti-Koski M, Puukka P, Kinnunen M, Jallinoja P, Absetz P. Effects of a healthy food supply intervention in a military setting: positive changes in cereal, fat and sugar containing foods. Int J Behav Nutr. 2012;9:91.

8. Nindl BC, Leone CD, Tharion WJ, Johnson RF, Castellani JW, Patton JF, et al. Physical performance responses during $72 \mathrm{~h}$ of military operational stress. Med Sci Sports Exerc. 2002;34:1814-22. 
9. Kivimäki M, Virtanen M, Elovainio M, Kouvonen A, Väänänen A, Vahtera J. Work stress in the etiology of coronary heart disease-a meta-analysis. Med Sci Sports Exerc. 2006;32:431-42.

10. Hoerster KD, Lehavot K, Simpson T, McFall M, Reiber G, Nelson KM. Health and health behavior differences: U.S. Military, veteran, and civilian men. Am J Prev Med. 2012;43:483-9.

11. Crum-Cianflone NF, Frasco MA, Armenta RF, Phillips CJ, Horton J, Ryan MA, et al. Prescription Stimulants and PTSD Among U.S. Military Service Members. J Trauma Stress. 2015;28:585-9.

12. Edmondson D, Kronish IM, Shaffer JA, Falzon L, Burg MM. Posttraumatic stress disorder and risk for coronary heart disease: a meta-analytic review. Am Heart J. 2013;166:806-14.

13. Stewart IJ, Sosnov JA, Howard JT, Orman JA, Fang R, Morrow BD, et al. Retrospective Analysis of Long-Term Outcomes After Combat Injury: A Hidden Cost of War. Circulation. 2015;132:2126-33.

14. Escott-Stump S, Mahan L, Raymond J. "Krause's food \& the nutrition care process." Missouri: Elsevier/Saunders; 2012. P,132-143.

15. Haffner SM. Pre-diabetes, insulin resistance, inflammation and CVD risk. Diabetes Res Clin Pract. 2003;61 Suppl 1:9-18.

16. Berr F, Goetz A, Schreiber E, Paumgartner G. Effect of dietary n-3 versus n-6 polyunsaturated fatty acids on hepatic excretion of cholesterol in the hamster. J Lipid Res. 1993;34:1275-84.

17. Hodge AM, English DR, O'Dea K, Sinclair AJ, Makrides M, Gibson RA, et al. Plasma phospholipid and dietary fatty acids as predictors of type 2 diabetes: interpreting the role of linoleic acid. Am J Clin Nutr. 2007;86:189-97.

18. Carroll RJ, Midthune D, Subar AF, Shumakovich M, Freedman LS, Thompson FE, et al. Taking advantage of the strengths of 2 different dietary assessment instruments to improve intake estimates for nutritional epidemiology. Am J Epidemiol. 2012;175:340-7.

19. Shim JS, Oh K, Kim HC. Dietary assessment methods in epidemiologic studies. Epidemiol Health. 2014;36:e2014009-

20. Cantwell MM. Assessment of individual fatty acid intake. Proc Nutr Soc. 2000;59:187-91.

21. Nascimento CM, Ribeiro EB, Oyama LM. Metabolism and secretory function of white adipose tissue: effect of dietary fat. An Acad Bras Cienc. 2009;81:453-66

22. Fernández-Real J-M, Vendrell J, Ricart W. Circulating adiponectin and plasma fatty acid profile. Clin Chem. 2005;51:603-9.

23. Kennedy A, Martinez K, Chuang C-C, LaPoint K, McIntosh M. Saturated fatty acid-mediated inflammation and insulin resistance in adipose tissue: mechanisms of action and implications. J Nutr. 2009;139:1-4.

24. Maeda N, Takahashi M, Funahashi T, Kihara S, Nishizawa H, Kishida K, et al. PPARy ligands increase expression and plasma concentrations of adiponectin, an adipose-derived protein. Diabetes. 2001;50:2094-9.

25. Bradley RL, Fisher FM, Maratos Flier E. Dietary Fatty Acids Differentially Regulate Production of TN-a and IL-10 by Murine 3T3-L1 Adipocytes. 2008;16:938-44.

26. Enzenbach C, Kröger J, Zietemann V, Jansen EH, Fritsche A, Döring F, et al. Erythrocyte membrane phospholipid polyunsaturated fatty acids are related to plasma C-reactive protein and adiponectin in middle-aged German women and men. Eur J Nutr. 2011;50:625-36.

27. Martin H. Role of PPAR-gamma in inflammation. Prospects for therapeutic intervention by food components. Mutat Res Fund Mol M. 2010;690:57-63.

28. Fritsche KL. Too much linoleic acid promotes inflammation-doesn't it? Prosta Leukot. Essent Fatty Acids. 2008;79:173-5.

29. Kong A, Neuhouser ML, Xiao L, Ulrich CM, McTiernan A, Foster-Schubert KE. Higher habitual intake of dietary fat and carbohydrates are associated with lower leptin and higher ghrelin concentrations in overweight and obese postmenopausal women with elevated insulin levels. Nutr Res. 2009;29:768-7

30. Arab L, Akbar J. Biomarkers and the measurement of fatty acids. Public health nutr. 2002;5:865-71.

31. Arab L. Biomarkers of fat and fatty acid intake. Health. Nutr. 2003;133 Suppl 3:925-32.

32. Jump DB. Dietary polyunsaturated fatty acids and regulation of gene transcription. Curr Opin Lipidol. 2002;13:155-64.

33. Field FJ, Born E, Mathur SN. Fatty acid flux suppresses fatty acid synthesis in hamster intestine independently of SREBP-1 expression. $J$ Lipid Res. 2003;44:1199-208.

34. Xu J, Nakamura MT, Cho HP, Clarke SD. Sterol regulatory element binding protein-1 expression is suppressed by dietary polyunsaturated fatty acids a mechanism for the coordinate suppression of lipogenic genes by polyunsaturated fats. J Biol Chem. 1999;274:23577-83.

35. Fernandez ML, McNamara DJ. Dietary fat-mediated changes in hepatic apoprotein BE receptor in the guinea pig: Effect of polyunsaturated, monounsaturated, and saturated fat. 1089;38:1094-102.

36. Tripodi A, Loria P, Dilengite M, Carulli N. Effect of fish oil and coconut oil diet on the LDL receptor activity of rat liver plasma membranes. Biochim Biophys Acta Lipids Lipid Metab. 1991;1083:298-30

37. Fernandez ML, Lin E, McNamara DJ. Differential effects of saturated fatty acids on low density lipoprotein metabolism in the guinea pig. $J$ Lipid Res. 1992;33:1833-42.

38. Tobin KAR, Steineger HH, Alberti S, Spydevold $\emptyset$, Auwerx J, Gustafsson J-Ak, et al. Cross-talk between fatty acid and cholesterol metabolism mediated by liver $X$ receptor-a. Mol Endocrinol. 2000;14:741-52.

39. Gillingham LG, Gustafson JA, Han S-Y, Jassal DS, Jones PJ. High-oleic rapeseed (canola) and flaxseed oils modulate serum lipids and inflammatory biomarkers in hypercholesterolaemic subjects. Br J Nutr. 2011;105:417-27.

40. Adams TH, Walzem RL, Smith DR, Tseng S, Smith SB. Hamburger high in total, saturated and trans-fatty acids decreases HDL cholesterol and LDL particle diameter, and increases TAG, in mildly hypercholesterolaemic men. Br J Nutr. 2010;103:91-8. 
41. Singh RB, Dubnov G, Niaz MA, Ghosh S, Singh R, Rastogi SS, et al. Effect of an Indo-Mediterranean diet on progression of coronary artery disease in high risk patients (Indo-Mediterranean Diet Heart Study): a randomised single-blind trial. Lancet. 2002;360:1455-61.

42. Worgall TS, Sturley SL, Seo T, Osborne TF, Deckelbaum RJ. Polyunsaturated fatty acids decrease expression of promoters with sterol regulatory elements by decreasing levels of mature sterol regulatory element-binding protein. J Biol Chem. 1998;273:25537-40.

43. Retzlaff BM, Walden CE, Dowdy AA, McCann BS, Anderson K, Knopp R. Changes in plasma triacylglycerol concentrations among free-living hyperlipidemic men adopting different carbohydrate intakes over 2 y: the Dietary Alternatives Study. Am J Clin Nutr. 1995;62:988-

44. Harbis A, Defoort C, Narbonne H, Juhel C, Senft M, Latgé C, et al. Acute hyperinsulinism modulates plasma apolipoprotein b-48 triglyceride-rich lipoproteins in healthy subjects during the postprandial period. Diabetes. 2001;50:462-9.

45. Kinosian B, Glick H, Preiss L, Puder KL. Cholesterol and coronary heart disease: Predicting risks in men by changes in levels and ratios. $J$ Investig Med. 1995;43:443-50.

46. Truswell A. Cereal grains and coronary heart disease. Eur J Clin Nutr. 2002;56:1-14.

47. Morgan L, Tredger J, Shavila Y, Travis J, Wright J. The effect of non-starch polysaccharide supplementation on circulating bile acids, hormone and metabolite levels following a fat meal in human subjects. Br J Nut. 1993;70:491-501.

48. Kelly SA, Frost G, Whittaker V, Summerbell CD. Low glycaemic index diets for coronary heart disease. Cochrane Database Syst Rev . 2004;4:CD004467

49. Esteghamati A, Meysamie A, Khalilzadeh O, Rashidi A, Haghazali M, Asgari F, et al. Third national Surveillance of Risk Factors of Non-Communicable Diseases (SuRFNCD-2007) in Iran: methods and results on prevalence of diabetes, hypertension, obesity, central obesity, and dyslipidemia. BMC Public Health. 2009;9:167.

50. Cloeren M, Mallon TM. Managing workers' compensation costs in the military setting: the Army's story. Clin Occup Environ Med. 2004;4:323-39.

\section{Supplementary Files}

This is a list of supplementary files associated with this preprint. Click to download.

- 4FFQE.docx

- DailyMinerallntake.docx

- DailyVitaminsIntake.docx 